\title{
Modeling time taken for HIV testing and visits in follow-up clinic to uptake test results: an application of extended Cox proportional hazard model
}

\author{
Suchira S.S. Mullege ${ }^{1}$, Samita S. ${ }^{2}$ \\ ${ }^{1}$ South Asian Regional Office, International Planned Parenthood Federation \\ ${ }^{2}$ University of Peradeniya, Sri Lanka
}

\begin{abstract}
Introduction: Improving human immunodeficiency virus (HIV) testing among most at risk populations (MARP) is one of the first steps to achieve sustainable development goal target of ending acquired immunodeficiency syndrome (AIDS) by 2030. Studying the time taken for HIV testing and subsequent clinic visits to collect the results provide important inputs for development of HIV prevention programs. This study aimed to identify the pattern of HIV testing behavior of female sex workers (FSWs) over time and developing of statistical models to describe HIV testing behavior among MARP.

Material and methods: HIV testing data of 5,667 FSWs registered with national HIV prevention program in 10 districts of Sri Lanka during 2016 and 2017 were analyzed using Cox proportional hazard model (CPHM) with time-dependent covariates.

Results: Time taken to acceptance of HIV testing is more dependent on individual factors of FSWs, whereas the uptake of results is less dependent on individual factors. Youth FSWs take more time to accept an HIV test. FSWs who operate in districts out of Colombo, use condoms and report undergoing HIV testing before joining the programme take more time for testing and collection of the results. FSWs with high partner exchange rate (therefore, at high-risk) are less likely to complete both events and take more time for testing. Most of these covariates were identified as time-dependent, and the effects showed a reducing pattern over time. Extended CPHM paved the way to model such variables.

Conclusions: Factors identified in the study, as associated with time taken for both events, can be consider in planning activities leading to HIV preventions programs. Extended CPHM was found to be an alternative technique to CPHM to model time for testing and subsequent clinic visit to uptake the results, especially in the presence of an HIV prevention program.
\end{abstract}

HIV AIDS Rev 2020; 19, 3: 157-166 DOI: https://doi.org/10.5114/hivar.2020.99616

Key words: HIV testing, uptake of result, survival modelling, extended Cox proportional hazard model, time-dependent covariates.

Address for correspondence: Suchira S.S. Mullege, Senior Technical Advisor (Organizational Learning and Evaluation), International Planned Parenthood Federation (South Asian Region), 37/27, Bullers' Lane, Colombo - 07, Sri Lanka, e-mail: suchirasuranga@gmail.com
Article history:

Received: 03.12.2019

Received in revised form: 23.02.2020

Accepted: 03.03.2020

Available online: 30.08 .2020
International Journal of HIV-Related Problem

HIV \& AIDS

R e v i e w 


\section{Introduction}

Globally, all the nations are intensely working to realize the sustainable development goal of ending the acquired immunodeficiency syndrome (AIDS) epidemic by 2030 [1]. Crucial parts of this plan are early detection of human immunodeficiency virus (HIV) cases and HIV treatment delivery to all in need. The 90-90-90 concept is one element of this plan [2]. As per the $90-90-90$ targets, (1) by $2020,90 \%$ of all people living with HIV (PLHIV) will know their HIV status, (2) by $2020,90 \%$ of all people with diagnosed HIV infection will receive sustained antiretroviral therapy, and (3) by $2020,90 \%$ of all people receiving antiretroviral therapy will have viral suppression [2]. Therefore, improving HIV testing among MARP is the first step to achieve the 90-90-90 targets. Researches indicate that by 2012 , less than $50 \%$ of MARP in many Asian and African countries did not know their HIV status [2]. In Sri Lanka, only 77\% of PLHIV know their HIV status [3]. Therefore, it is important that MARP undergo HIV testing periodically to know their HIV status. It usually takes a few days to a few weeks to get results of an HIV test; although, there are some rapid expensive HIV tests, which can produce results within 30 minutes. Therefore, follow-up clinic visits to collect the results is also important to know HIV status [4]. Various studies have indicated that nearly one third of patients screened for HIV by traditional programs, with pretest counseling and blood tests that are sent to a central laboratory, fail to collect the results [5].

The 90-90-90 targets cannot be achieved without identifying and overcoming the factors that influence undergoing HIV testing by MARP. Moreover, studying the pattern of HIV testing over time and modeling covariates with time taken to HIV testing will provide important inputs for development of HIV prevention programs. Further, understanding both HIV testing behavior and subsequent uptake of results are equally important for designing HIV prevention programs. Previous studies primarily focused on either completion of the event without evaluating the time factor or considered only HIV testing, but not the collection of results [6-9].

The aim of this study was to understand the pattern of HIV testing among MARP over time, considering HIV testing and collection of results as two ordered events, and to identify suitable technique to model those time events, especially in the presence of time-dependent factors. Female sex workers (FSWs) who reported selling sex in exchange for money or goods during last 12 months in 10 districts of Sri Lanka were selected as the MARP sample in the study.

\section{Material and methods}

The study was conducted using a web-based data source known as Monitoring and Evaluation Information Management System (MEIMS), maintained by the Family Planning Association of Sri Lanka (FPASL) in line with the National HIV Prevention Program during 2016 and 2017. The database holds the capacities for data filtration and export [10].

\section{Background of the data}

Data from MEIMS on client registration and HIV testing were used for the study. At the time of registration, each FSW receives a unique identification/registration number, which is used to identify the person throughout the project time. The client registration form contains FSW registration number, socio-demographic data, data on HIV risk behaviors, and health seeking behaviors. Although the personal information of clients has been recorded in the client registration form to ensure the continuity of care and follow-up services, as per the national guideline, the personal information is not accessible in the electronic database to maintain the confidentiality of information [10]. The client registration data for MEIMS are supported by trained project coordinators of community-based organization $(\mathrm{CBO})$ at the district level, using the client registration forms completed by field supervisors (FS) and peer educators (PE). These data are secondarily verified by the monitoring and evaluation staff in the project to to ensure data quality [10].

FSWs who are registered with the program are escorted to nearby sexually transmitted disease (STD) clinics, located in government hospitals. HIV testing data, which are available in MEIMS are captured using clinics' referral slips issued by STD clinics. The monitoring and evaluation staff at the Family Planning Association verify the slips for accuracy and consistency, and the data is entered into MEIMS [10].

\section{Sampling and inclusion criteria}

The FSWs registered with the national HIV prevention program in 10 administrative districts (Anuradhapura, Colombo, Galle, Gampaha, Hambantota, Kandy, Kurunegala, Matara, Polonnaruwa, and Ratnapura) were considered for this study (Table 1). There was no sample size calculation involved, as all the FSWs who received services from the project were studied retrospectively. However, the FSW who received services less than six months (loss to follow-up before completion of first six months) were excluded from the study. The study period was January 1, 2016 to December 31, 2017.

\section{Ethical concerns}

As per the monitoring and evaluation policy of FPA Sri Lanka, the executive director is accountable for correct use of all monitoring and evaluation information available in MEIMS [11]. The executive director granted permission to use HIV testing data to perform this study. For ethical approval, the research protocol with other related documents were submitted to the ethical review committee (ERC) of the Sri Lanka Medical Association (SLMA). The ethical approval was received on April 20 2018.

\section{Cox proportional hazard model}

CPHM is a survival model introduced by Cox in 1972, who extended the method of non-parametric Kaplan-Meier 
estimates to regression type arguments for life-table analyses. Cox advanced the prediction of survival time in individual subjects by utilizing variables covarying with survival and ignoring the baseline hazard of individuals. In this model, no assumptions about the baseline hazard of individuals are made, and only presumed that the hazard functions of different individuals remain proportional and constant over time [12]. The Cox regression model has been used in many fields, including HIV $[13,14]$. The idea of this model is to define the hazard level as a dependent variable, which is explained by the time-related component (so-called, baseline hazard) and the covariates-related component [15]. The model is defined as follows:

$$
\lambda(t, \boldsymbol{x})=\lambda_{0}(t) \exp (\beta \boldsymbol{x})
$$

where $\lambda(t, x)$ is the hazard function that depends on time point $t$ and vector of covariates $x, \lambda_{0}(t)$ is the baseline hazard function that depends on time only, and $\exp (\beta \boldsymbol{x})$ is the covariates-related component.

Many authors have discussed the importance of proportional hazard assumption and suggested potential modification of this model if the hazard ratio turns out not to be constant over time for some covariates [16]. If the influence differs over time and this changing impact is also of interest, then one of the available methods of Cox model modification for non-proportional hazards might be applied. Including the interaction with time in the model enables interpretation of the parameters, because the covariate's influence at the hazard level is not constant [15].

If continuous variable $\mathrm{A}$ does not depend on time, and the factor B is time-dependent with two levels, 1 and 2, the hazard ratio HR between level 1 and level 2 of factor B is given by:

$H R=\frac{\lambda(t, A=a, B=2)}{\lambda(t, A=a, B=1)}=\frac{\lambda_{0} \exp \left(\beta_{1} a+2 \beta_{2}+2 \beta_{3} t\right)}{\lambda_{0} \exp \left(\beta_{1} a+\beta_{2}+\beta_{3} t\right)}=\exp ^{\beta_{2}+\beta_{3} t}$,

where $\beta_{1}$ is the parameter estimate for variable $\mathrm{A}, \beta_{2}$ is the parameter estimate for variable $\mathrm{B}$, and $\beta_{3}$ is the parameter estimate for interaction of variable $\mathrm{A}$ and time $(t)$.

Therefore, the hazard ratio of time-dependent covariates is changing over time and the interpretation needs to be performed accordingly [15].

\section{Data analysis}

In this study, as illustrated in Figure 1, two events can be defined, including (1) testing for HIV (collection of blood sample) for the first time after registration with the program ("testing" in this paper), and (2) subsequent clinic visit to receive HIV test results ("uptake of results" in this paper).

As most of STD clinics do not perform rapid HIV test, the results cannot be handed over within the same day. Therefore, each subject (i.e., FSW) will have to undergo both events in two-time span. Considering the nature of these two events
Table 1. Number of female sex workers included in the study differentiated by district

\begin{tabular}{l|c|c|c}
\hline District & Frequency & Percentage & $\begin{array}{c}\text { Cumulative } \\
\text { percentage }\end{array}$ \\
\hline Anuradhapura & 156 & 2.8 & 2.8 \\
\hline Colombo & 2,535 & 44.7 & 47.5 \\
\hline Galle & 374 & 6.6 & 54.1 \\
\hline Gampaha & 1,024 & 18.1 & 72.2 \\
\hline Hambantota & 83 & 1.5 & 73.6 \\
\hline Kandy & 511 & 9.0 & 82.6 \\
\hline Kurunegala & 189 & 3.3 & 86.0 \\
\hline Matara & 237 & 4.2 & 90.2 \\
\hline Polonnaruwa & 255 & 4.5 & 94.7 \\
\hline Ratnapura & 303 & 5.3 & 100.0 \\
\hline Total & 5,667 & 100.0 & \\
\hline
\end{tabular}

that the second event cannot take place without the first event, these types of events are called "ordered events".

In survival analysis, the outcome variable is the time to event (called "survival time"), which can be measured in days, weeks, years, etc. Since the current study is focusing on two ordered events, three survival times can be defined, such as (1) number of days from registration to testing $\left(\mathrm{T}_{1}\right)$, (2) number of days from testing to the uptake of results $\left(\mathrm{T}_{2}\right)$, and (3) number of days from registration to the uptake of results $\left(\mathrm{T}_{3}=\mathrm{T}_{1}+\mathrm{T}_{2}\right)$. Three survival times were considered one at a time, which resulted following three CPHMs.

The statistical software SAS university edition was used for univariate and multivariate modelling. The PHREG procedure of SAS software was adopted. All the models were initially developed without considering the time dependency and tested for proportional hazard assumption. As the proportional hazard assumption was violated for some of the covariates of three initial models, the time-dependent covariates (interaction of covariates with time) were

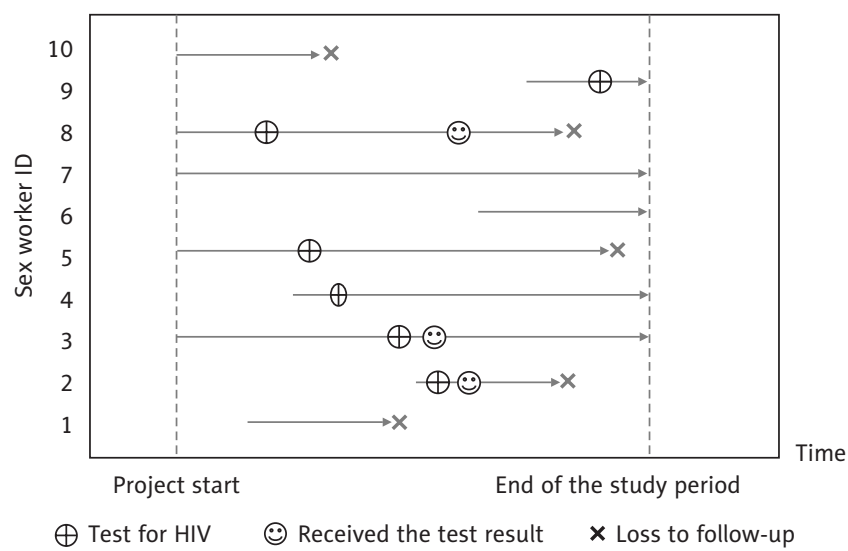

Figure 1. Graphical representation of HIV testing behavior of female sex workers 
Table 2. Sample characteristics

\begin{tabular}{|c|c|c|c|}
\hline Variable/Levels & Frequency & Percentage & $\begin{array}{l}\text { Cumulative } \\
\text { percentage }\end{array}$ \\
\hline \multicolumn{4}{|l|}{ Location } \\
\hline Rural & 669 & 11.8 & 11.8 \\
\hline Semi urban & 1,492 & 26.3 & 38.1 \\
\hline Urban & 3,506 & 61.9 & 100.0 \\
\hline Total & 5,667 & 100.0 & \\
\hline \multicolumn{4}{|l|}{ Age } \\
\hline Below 25 & 382 & 6.8 & 6.8 \\
\hline 25 and above & 5,269 & 93.2 & 100.0 \\
\hline Total & 5,651 & 100.0 & \\
\hline \multicolumn{4}{|l|}{ Level of education } \\
\hline Up to grade 9 & 3,083 & 54.4 & 54.4 \\
\hline Grade 10 and above & 2,584 & 45.6 & 100.0 \\
\hline Total & 5,667 & 100.0 & \\
\hline \multicolumn{4}{|l|}{ Marital status } \\
\hline Never married & 398 & 7.1 & 7.1 \\
\hline Married & 3,610 & 64.8 & 71.9 \\
\hline Living together & 436 & 7.8 & 79.7 \\
\hline Separated & 206 & 3.7 & 83.4 \\
\hline Divorced & 405 & 7.3 & 90.7 \\
\hline Widow & 520 & 9.3 & 100.0 \\
\hline Total & 5,575 & 100.0 & \\
\hline \multicolumn{4}{|l|}{ FSW typology } \\
\hline Based in street own house or slums & 3,291 & 59.9 & 59.9 \\
\hline Based in lodge, hotel, brothel, or restaurant & 604 & 11.0 & 70.9 \\
\hline Based in massage center & 1,145 & 20.8 & 91.7 \\
\hline Other & 456 & 8.3 & 100.0 \\
\hline Total & 5,496 & 100.0 & \\
\hline
\end{tabular}

included in the model. The information criteria of new models were much lower than the initial models indicating good model fitting. Both, the log transformation of the time variable and the time variable itself were included in model statement, one at a time. The log time transformation was used in the final models, since it resulted comparatively better model fit. The extended CPHMs were used as the final models for interpretation.

\section{Results}

After data cleaning and checking for exclusion criteria (FSWs who have not completed minimum six months with the project), the total of 5,667 FSWs were included in the study. The composition of the sample is presented in Table 2. The majority was from Colombo district, followed by Gampaha district. The size of sample from selected districts was aligned with the estimated percentage of FSWs in each district of Sri Lanka [17]. Majority (62\%) of FSWs in the sample operated in urban settings. Around $7 \%$ of FSWs were young below 25 years of age. Almost half of participants $(46 \%)$ were educated and completed more than 8 years in school. Majority of FSWs (85\%) were ever married, of which $65 \%$ were currently married, and $7 \%$ were never married FSWs in the sample. Most of FSWs (60\%) were operated in very informal settings, such as streets, own houses, or slums. Around one fifth of the participants $(21 \%)$ were based in massage centers. Only around one tenth of the FSWs (11\%) were based in lodges, hotels, brothels, or restaurants.

\section{Model $01-\mathrm{T}_{1}$}

The parameters estimate of the Cox regression model are given in Table 3. The parameter estimates are the log hazard ratios, and hazard ratios are the relative risks. The estimate 
Table 3. Results of extended CPHMs with time-dependent covariates

\begin{tabular}{|c|c|c|c|c|c|c|c|c|}
\hline & & & \multirow{2}{*}{\multicolumn{2}{|c|}{$\begin{array}{c}\text { Model } 01-\mathrm{T}_{\mathbf{1}} \\
37319.117\end{array}$}} & \multicolumn{2}{|c|}{ Model $02-\mathrm{T}_{2}$} & \multicolumn{2}{|c|}{ Model $03-\mathrm{T}_{3}$} \\
\hline \multirow{3}{*}{ Model fit statistics } & \multicolumn{2}{|c|}{$-2 \log L$} & & & \multicolumn{2}{|c|}{23130.785} & \multicolumn{2}{|c|}{25653.465} \\
\hline & \multicolumn{2}{|c|}{ AIC } & \multicolumn{2}{|c|}{37337.117} & \multicolumn{2}{|c|}{23146.785} & \multicolumn{2}{|c|}{25667.465} \\
\hline & \multicolumn{2}{|c|}{ SBC } & \multicolumn{2}{|c|}{37388.965} & \multicolumn{2}{|c|}{23189.777} & \multicolumn{2}{|c|}{25705.083} \\
\hline \multicolumn{9}{|c|}{ Effects } \\
\hline \multicolumn{2}{|l|}{ Variable } & Level & Hazard ratio & $\operatorname{Pr}>\chi^{2}$ & Hazard ratio & $\operatorname{Pr}>\chi^{2}$ & Hazard ratio & $\operatorname{Pr}>\chi^{2}$ \\
\hline \multicolumn{2}{|c|}{ Age (age in number of years) } & & 1.007 & 0.0072 & & & & \\
\hline \multicolumn{2}{|c|}{ District ( $1=$ Colombo, 2 = other) } & 2 & 0.880 & 0.0040 & 0.869 & 0.0084 & 0.839 & 0.0013 \\
\hline \multicolumn{2}{|c|}{ Number of sexual partners } & & & & 0.981 & 0.0002 & 0.881 & $<0.0001$ \\
\hline \multicolumn{2}{|c|}{$\begin{array}{l}\text { HIV testing }(0=\text { not subjected for HIV } \\
\text { test during past year, } 1=\text { subjected for } \\
\text { HIV test during past year) }\end{array}$} & 1 & 0.375 & $<0.0001$ & & & 0.130 & $<0.0001$ \\
\hline \multicolumn{2}{|c|}{$\begin{array}{l}\text { Condom use }(0=\text { not used condoms } \\
\text { during last sexual encounter, } 1=\text { used } \\
\text { condoms during last sexual encounter })\end{array}$} & 1 & 0.364 & $<0.0001$ & 0.621 & $<0.0001$ & 0.226 & $<0.0001$ \\
\hline \multirow{2}{*}{\multicolumn{2}{|c|}{$\begin{array}{l}\text { Typology }(1=\text { based in street own } \\
\text { house or slums, } 2=\text { based in hotel } \\
\text { lodge, brothel, or restaurant, } 3=\text { based } \\
\text { in massage center) }\end{array}$}} & 2 & 1.968 & $<0.0001$ & 0.640 & 0.0258 & 1.407 & 0.0727 \\
\hline & & 3 & 4.746 & $<0.0001$ & 0.485 & $<0.0001$ & 3.392 & 0.0002 \\
\hline \multicolumn{9}{|l|}{ Contrasts (typology) } \\
\hline \multicolumn{2}{|l|}{ Typology 1 vs. 2} & & 0.508 & $<0.0001$ & & & 0.711 & n.s. \\
\hline \multicolumn{2}{|l|}{ Typology 1 vs. 3} & & 0.211 & $<0.0001$ & & & 0.295 & $<0.0001$ \\
\hline \multicolumn{2}{|l|}{ Typology 2 vs. 3} & & 0.415 & $<0.0001$ & & & 0.415 & $<0.0001$ \\
\hline \multicolumn{9}{|l|}{ Interaction effect } \\
\hline \multirow{2}{*}{\multicolumn{2}{|c|}{ Condom use * typology }} & $1 / 2$ & & & 1.343 & 0.1794 & & \\
\hline & & $1 / 3$ & & & 2.170 & $<0.0001$ & & \\
\hline \multicolumn{9}{|c|}{ Contrasts (condom use * typology) } \\
\hline Condom use 0 vs & $y=1$ & & & & 1.609 & $<0.0001$ & & \\
\hline Condom use 0 vs & $y=2$ & & & & 1.198 & n.s. & & \\
\hline Condom use 0 vs & $y=3$ & & & & 0.742 & $<0.0001$ & & \\
\hline Time-dependent cov & & & & & & & & \\
\hline Typology*log (tim & & & 0.882 & $<0.0001$ & & & 0.914 & 0.0045 \\
\hline Condom use ${ }^{*} \log$ & & & 1.210 & $<0.0001$ & & & 1.294 & 0.0002 \\
\hline HIV testing ${ }^{*} \log (t$ & & & 1.148 & $<0.0001$ & & & 1.355 & $<0.0001$ \\
\hline Sexual partners* & & & & & 1.005 & 0.0017 & 1.022 & $<0.0001$ \\
\hline
\end{tabular}

n.s. - not significant

of age was 0.00649 and hence, hazard ratio of testing for one additional year of age was $\mathrm{e}^{0.00649}=1.007$. Therefore, we concluded that there was a 7\% higher chance of testing when the age was increased by 10 years. Youth FSWs have been more reluctant for testing compared to adults. Similarly, there was a $12 \%$ higher chance of testing among FSWs who were based in Colombo district compared to other districts.

Other covariates (testing for HIV before joining the program, use of condoms during the last sex at the time of registration, and FSW typology) were found to be time-dependent covariates and thus, the hazard ratio related to those factors changed over time.
As expected, HIV testing within 12 months prior to joining the program showed a negative association with HIV testing from the program. The FSWs who have not undergone testing before joining the program showed a $63 \%$ higher chance of testing from the program compared to the FSWs who were already tested for HIV.

As shown in Figure 2, this effect of testing before joining the program decreased over time, but it was significant even after two years. By the end of 600 days, the chance of testing was $45 \%$ higher among those FSWs who have not undergone testing within 12 months prior to joining the program. 


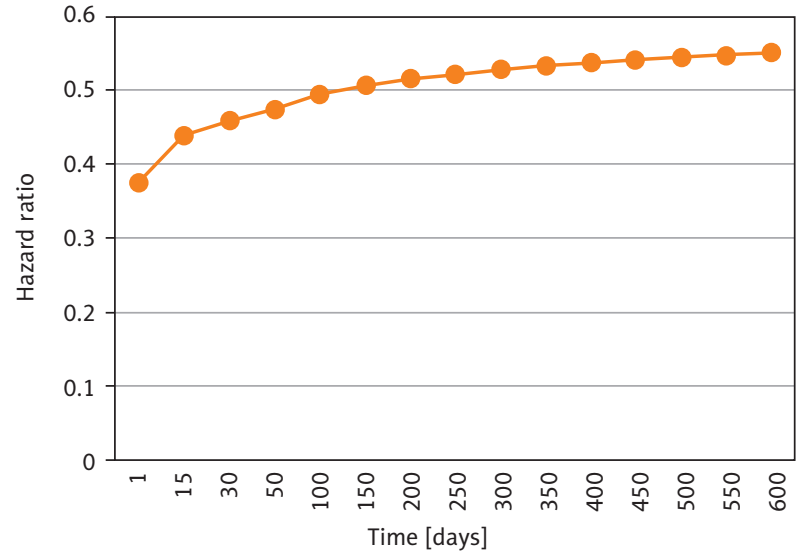

Figure 2. Time dependency of covariates. Graphical illustration of hazard ratio for HIV testing over time

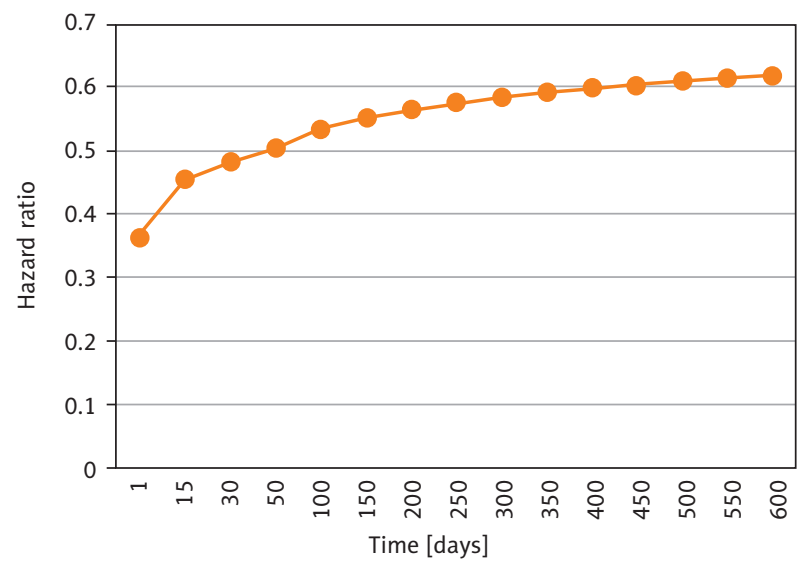

Figure 3. Time dependency of covariates. Graphical illustration of hazard ratio for condom use over time

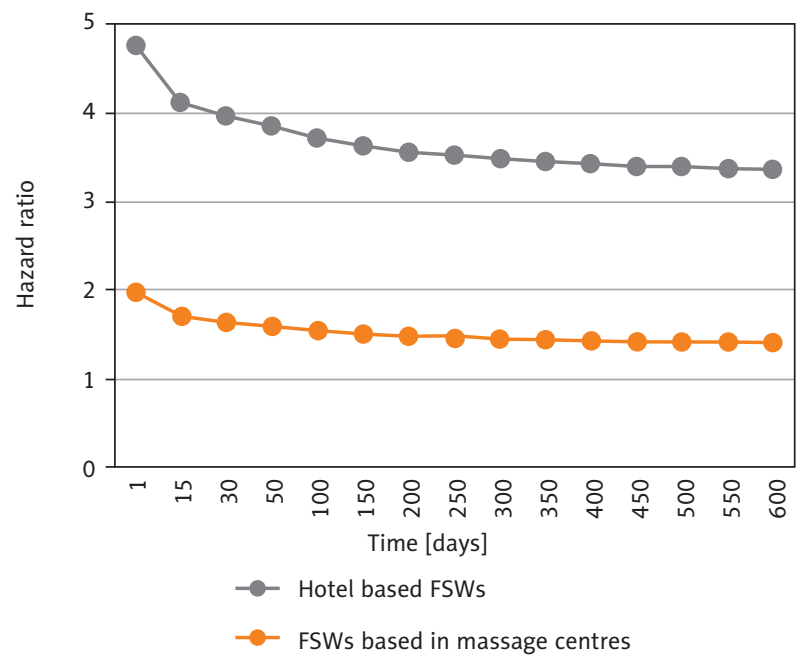

Figure 4. Time dependency of covariates and graphical illustration of hazard ratio for female sex workers (FSWs) typology over time. Street-based FSWs were considered as the reference group and were not included in the graph
Self-reported condom use during the last sex at the time of registration showed a similar effect on testing. FSWs who did not use condoms showed $64 \%$ higher chance of testing. This may be due to perceived low-risk of HIV infection among condom users. However, as illustrated in Figure 3, this effect decreased over time up to $38 \%$ by the end of two years. It can be assumed that some FSWs who did not use condoms at the time of registration may started using condoms due to behavioral change communication (BCC) interventions of the program. However, even at the end of the program, the testing was not similar between the two groups. Further research is needed to identify the reasons for this relationship between HIV testing and condom use.

FSW typology showed a greater association with testing. FSWs based in massage centers showed the lowest median time to test (369 days), followed by FSWs based in hotels (530 days) and street-based FSWs (569 days). FSWs who were based in massage centers showed almost 4 times (375\%) higher acceptance of HIV test compared to streetbased FSWs. The tendency of HIV testing by FSWs based in hotels or lodges were 97\% higher than street-based FSWs. Figure 4 shows the variability of hazard ratio over time considering street-based sex workers as the reference group. This HIV testing gap between the two groups indicated a declining trend over time. For example, HIV testing difference among street sex workers and sex workers based in massage centers declined from $375 \%$ to $235 \%$ by the end of the first 600 days. This reduction in time to test could be largely attributed to the effect of prevention program. However, this effect was significant among all three groups, even at the end of the program.

It is important to note that the interaction effect between FSW typology and condom use was significant in the initial model. However, it became insignificant after time-dependent covariates were introduced.

\section{Model $02-\left(T_{2}\right)$}

As presented in Table 3, FSW typology, condom use during the last sexual encounter at the time of registration, number of sexual partners during the month prior, and the district were identified as covariates, which significantly affected subsequent clinic visit to collect the results. The uptake of results by FSWs from other districts was 13\% lower than FSWs based in Colombo. This may be due to the difference in quality of the program in different geographical locations and the difference in distance to the nearest STD clinic. As illustrated in Figure 5, the interaction effect between FSW typology and condom use was significant $(p<0.0001)$. The association between condom use and uptake of test result was found to be depended on the FSW typology. The street-based FSWs who reported not using condoms demonstrated $61 \%$ higher chance for uptake the test result. However, for the FSWs who were based in massage centers, the association showed an inverse pattern.

The FSWs who were based in massage centers and reported not using condoms, demonstrated $26 \%$ lower chance 
A

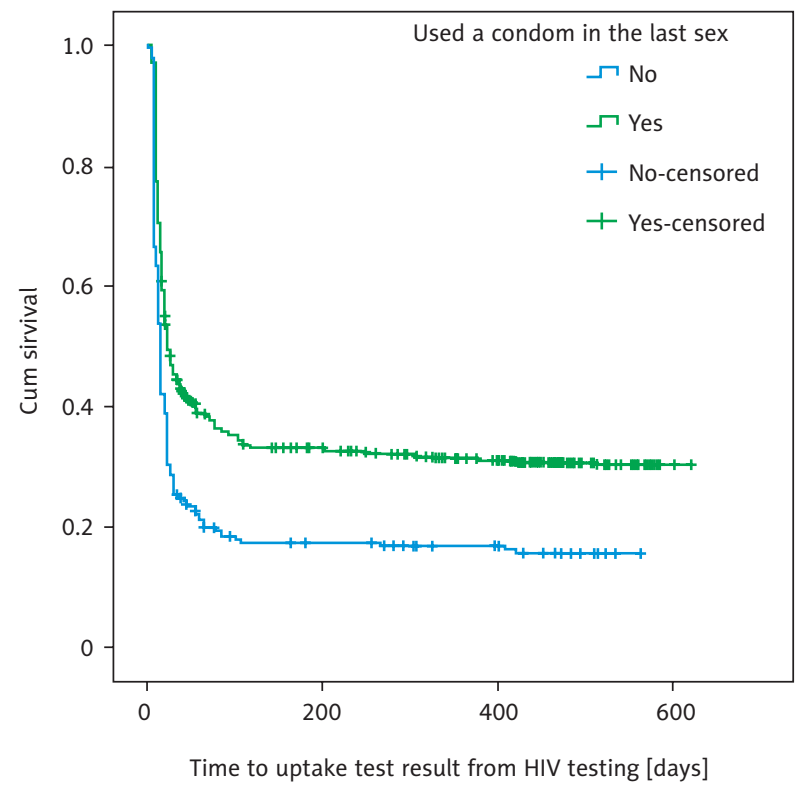

B

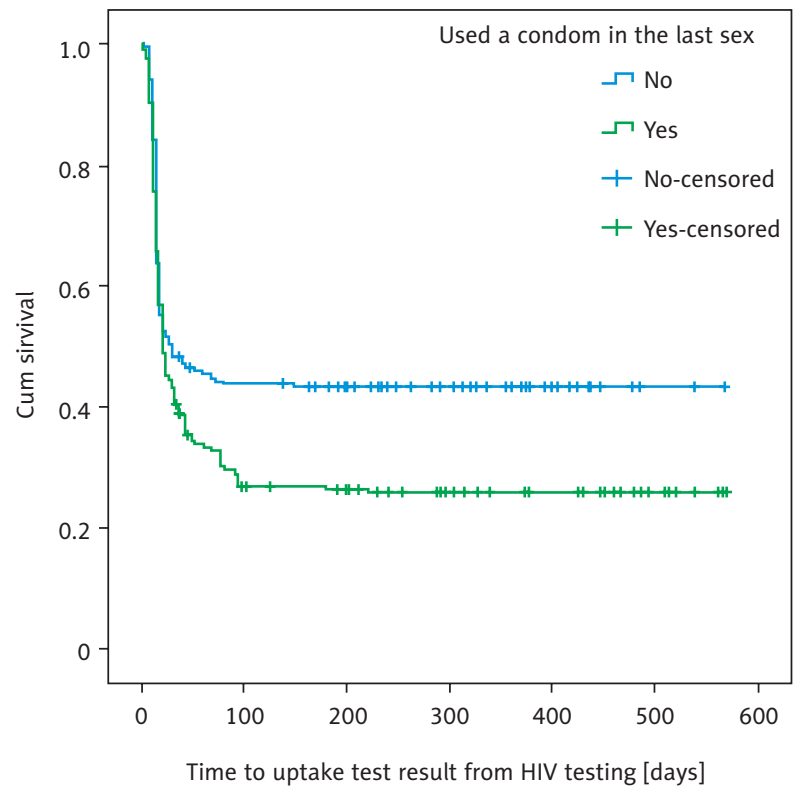

Figure 5. Graphical illustration of interaction effect between "female sex workers (FSWs) typology" and "condom use". A) Survival function of street based sex workers, B) survival function of the FSWs based in massage centers

for the uptake of results. The association between condom use and collection of results was not significant for those FSWs who were based in hotels and brothel houses.

As illustrated in Figure 6, the number of sexual partners during the past 30 days (partner exchange rate) was identified as the time-dependent covariate. Every additional sexual partner decreased the tendency to uptake the results by $2 \%$. However, this effect was declining over time up to $1 \%$ by the end of study period. It is important to note that high-risk FSWs are reluctant not only for testing, but also to collect the results, indicating the importance of pre-test counselling.

Most of the variables, which were significant in model 1 were not significant in model 2. Unlike the first visit to STD clinics for HIV testing, subsequent clinic visits to uptake the results may largely depend on the quality of facility and overall quality of care during the first visit, such as pre-test counselling, stigma and discrimination, time consumption, etc. However, these facility-related factors were not studied, as it was beyond the scope of this study. This could be the reason that only few individual factors were selected as covariates in the second model.

$$
\text { Model } 03-\mathrm{T}_{3}=\mathrm{T}_{1}+\mathrm{T}_{2}
$$

As elaborated in Table 3, except age, all other factors, which were included in model 1 and model 2 were significant in model 3. However, the interaction effect between typology and condom use was not significant in model 3. HIV testing, condom use, typology, and number of sexual partners were identified and included as time-dependent covariates in the model.

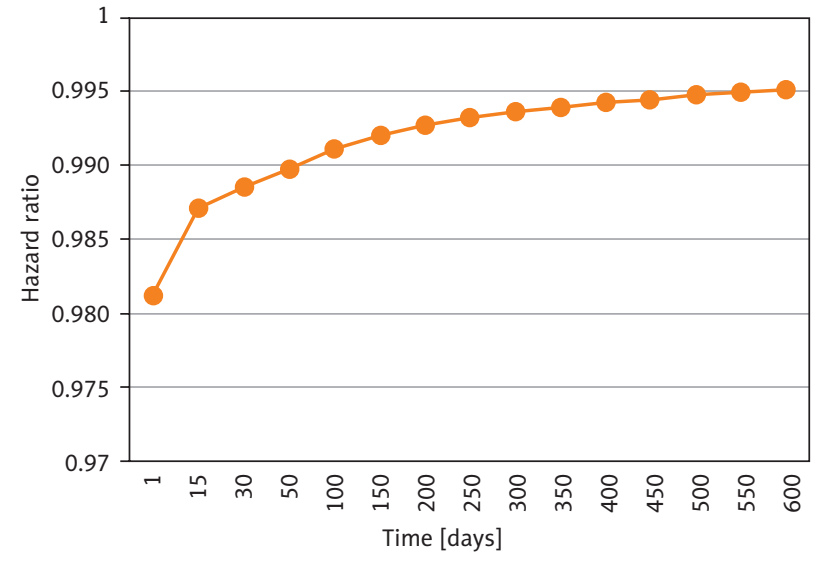

Figure 6. Time dependency of covariates. Graphical illustration of hazard ratio for number of sexual partners over time

The district, in which the FSWs were based was the only time-independent covariate. The FSWs based in other districts showed $17 \%$ lower tendency to complete both HIV events. This can be considered as an accumulated effect of the first event (12\%) and the second event (13\%).

Pattern of association with testing identified in model 1 was observed in model 3 (Figure 7). However, overall effect of testing for HIV before joining the project on HIV testing from the project, has improved after inclusion of the second event (although testing was not significant in the second model). FSWs who were tested for HIV during 12 months prior to registration demonstrated $87 \%$ lower chance of completing both events. However, this effect slowly declined up to $70 \%$ 


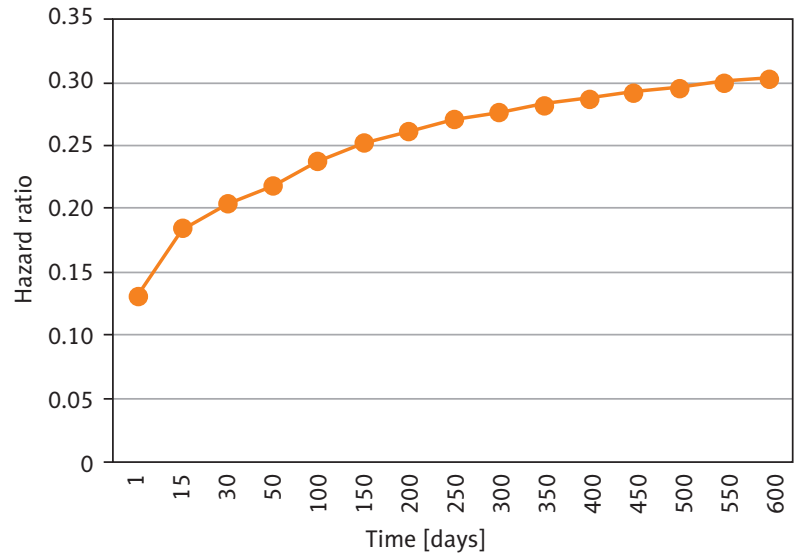

Figure 7. Time dependency of covariates. Graphical illustration of hazard ratio for HIV testing over time

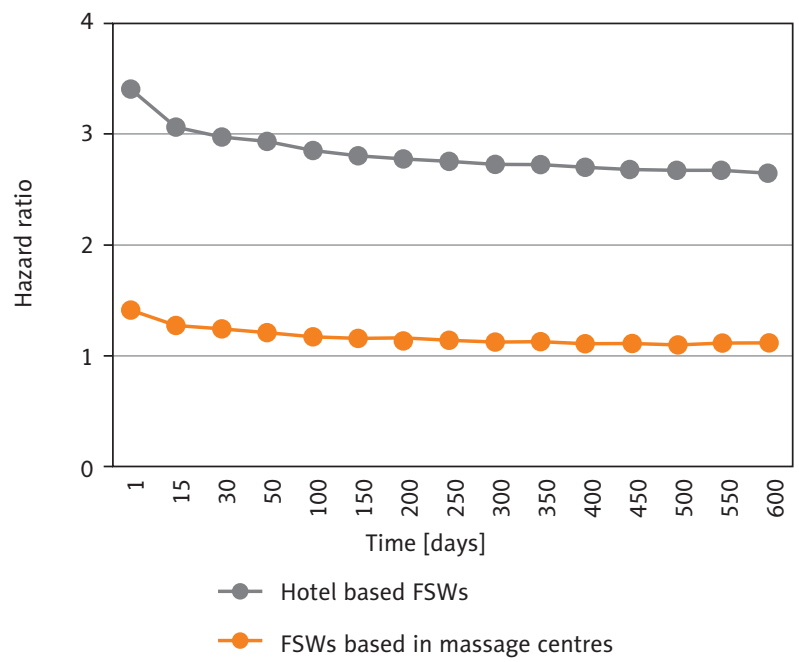

Figure 8. Time dependency of covariates. Graphical illustration of hazard ratio for female sex workers typology over time

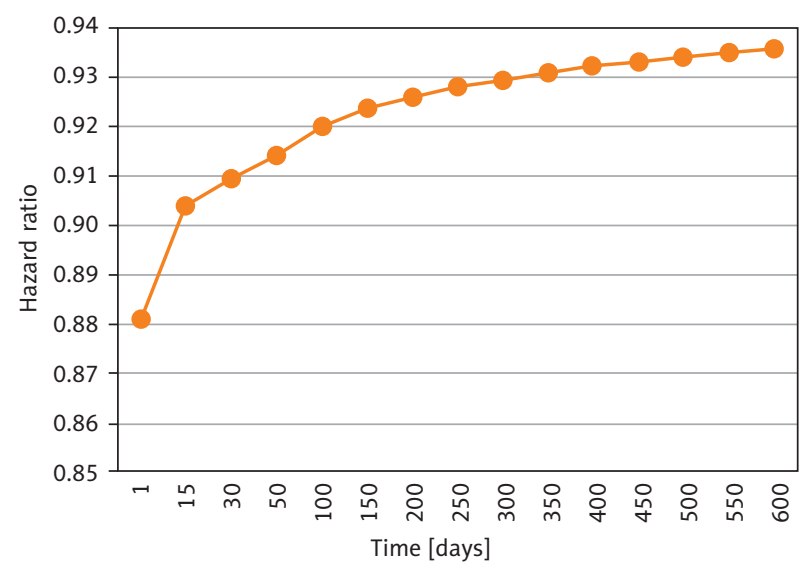

Figure 9. Time dependency of covariates. Graphical illustration of hazard ratio for the number of sexual partners over time during the first two years. The same pattern could be observed between condom users and non-users over time. The FSWs who have used a condom during the last sex at the time of registration showed $78 \%$ lower chance to complete both events. This effect of condom use before joining the project was reduced up to $54 \%$ by the end of the first 600 days.

Similar pattern of association between typology and HIV testing, which was observed in model 1 appeared also in model 3, but with relatively low intensity. In other words, an addition of survival time in the second event has lowered the overall effect of FSW typology. This may be due to the presence of interaction effect between the condom use and the typology in the second model.

FSWs who were based in massage centers showed 239\% higher chance for completing both events compared to street-based FSWs. However, there was no significant difference between street-based sex workers and FSWs based in hotels/ brothel houses. Chance of completion of both events by the FSW based in hotels or lodges were 59\% lower than the FSWs based in massage centers. Figure 8 shows the variability of hazard ratio over time considering street-based sex workers as the reference group. This difference of completing both events among the two groups indicated a declining trend over time. For instance, the difference among street sex workers and sex workers based in massage centers declined from $239 \%$ to $164 \%$ by the end of the first 600 days. The hazard ratio between street-based FSWs and FSWs based in massage centers approached 1 by the end of the project showing equal chance of completing both events (Figure 8).

The pattern of association between the number of sexual partners and the HIV testing over time, which we observed in model 2, was present also in model 3, but with a greater intensity (Figure 9). Every additional sexual partner decreased the tendency to complete both events by $12 \%$ at the beginning of the project. However, this effect declined over time up to $7 \%$ by the end of the project. The FSWs who were having high partner exchange rate (considered as highrisk for acquiring HIV) showed a lower chance of completing both events at the beginning of the project. However, this difference among two groups (high-risk and low-risk) indicated a declining trend over time. This may be because of program (BCC interventions), which can be considered as one of the particularly important finding of this study.

\section{Programme and policy implications}

Sri Lanka national HIV testing guidelines [18] clearly highlight that MARP including FSWs must test for HIV once in a six month (at least annual testing is promoted, based on partner exchange rate and safe sex practices). However, findings of this study demonstrated that testing for HIV within 12 months prior to joining the program significantly reduced testing and uptake of the results. This effect was significant even after two years and is assumed to be much higher among FSWs who were not covered by prevention interventions. This indicates a gap between the national target in terms of frequency in MARP testing and actual testing behavior. Future 
interventions must focus on increasing testing frequency among FSWs after controlling for the factors, which affect repeated testing.

The outcomes of this study demonstrated that testing was more dependent on individual factors of FSWs, whereas the uptake of results was less dependent on individual factors. A subsequent clinic visits to collect the results may be more dependent on the facility and service provider-related factors, such as personal experience of the first visit, effectiveness of pre-test counselling, stigma and discrimination, waiting time, etc. The time to uptake of results cannot be shortened without improving the quality standards of care.

It was evident that the testing behavior of the FSWs based in Colombo was significantly different from FSWs of other districts after controlling for all induvial socio-demographic and behavioral factors. The FSWs from other districts showed 13\% lower chance of testing and uptake the test results compared to the FSWs based in Colombo. The chance of loss to follow-up after the first clinic visit was also higher for those FSWs based in other districts. The FSWs who were based in other districts took relatively longer time for testing as well as for uptake of results. It is important to highlight the fact that the condom use also was lower among the FSWs based in other districts (68\%) compared to Colombo district (85\%). This may be due to early start of HIV prevention programs in Colombo district compared to other areas. Despite the differences, a uniform, not tailor-made prevention model is applied across Sri Lanka to suit all districts [19]. The results of this study highlight the requirement for development of different prevention strategies for districts outside Colombo. Current approach of implementation of uniform prevention model across the country needs to be revisited.

In this study, time to testing and uptake of results was clearly dependent on the FSW typology. Street-based FSWs and hotel-based FSWs were less likely to complete both events, compared to the FSWs based in massage centers with all other variables considered. This difference was found among those FSWs who reported using condoms during the last sex at the time of registration. The current HIV prevention program implements a uniform prevention model among all types of FSWs [19, 20]. Findings of this study clearly indicate the requirement for development and implementation of interventions considering the FSW typology.

Additionally, the results of the present study clearly emphasize that FSWs who used condoms were less likely to complete both events than FSWs who do not use condoms. The FSWs who use condoms took relatively longer time for testing and uptake of test results. This difference of HIV testing behavior among condom users and non-users was highest among streetbased FSWs, followed by hotel-based FSWs, and FSWs based in massage centers. This effect of condom use on testing was significant even after implementation of HIV prevention interventions for two years. This may be due to the perceived lowrisk among those FSWs who use condoms. The prevention programs should clearly communicate (FSWs) the fact that HIV testing at least once a year is important even if they use condoms consistently as condom use itself is not $100 \%$ protective.
Number of sexual partners (partner exchange rate) was one of the most important risk factors for HIV and other sexually transmitted infections (STI). This finding clearly indicates that the FSWs who were having higher partner exchange rate (therefore, at higher risk) were less likely to test for HIV and took longer time for testing. The chance of loss to follow-up after the first clinic visit was also higher among this high-risk FSWs. Therefore, it was evident that HIV-infected FSWs with high number of clients were operating for longer time without knowing their HIV status (not taking treatments) and transferred the disease to large number of clients. However, the FSWs with high partner exchange rate (above 20) were more likely to use condoms (90\%) than FSWs with less partner exchange rate (71\%). The current prevention model does not consider the risk profile of FSWs in planning and implementation. It is important to identify high-risk FSWs at the time of registration and provide a specific and more targeted prevention service package.

This model of HIV testing carried out in Sri Lanka was an incentive-driven model, where the peer educators who escort FSWs to STD clinics for testing receive a motivation (for both events). Although overall intervention clearly demonstrated a positive effect on HIV testing behavior of FSWs, the effect of incentive scheme was not studied in detail.

\section{Conclusions}

All three CPHMs generated consistent results, which can be used to describe the pattern of HIV testing and uptake of test result. Time to testing is more dependent on individual factors of FSWs, whereas time to uptake of results is less dependent on individual factors. Most of the factors were identified as time-dependent; therefore, the general CPHM must include time-dependent covariates in a model. As the study was carried out simultaneously with the national HIV prevention program, the time-dependent covariates may largely represent the effect of HIV prevention program. In other words, this approach can be used to measure the program effect; therefore, indirect and cost-effective method of program evaluation using secondary data. An extended CPHM is an effective and simple technique to model time to HIV testing and subsequent clinic visit to uptake the results of MARP.

\section{Conflicts of interest}

The authors declare no conflict of interest.

\section{References}

1. United Nations. Sustainable Development Goals - 17 Goals to Transform Our World. [Online] UN Web Services Section, Department of Public Information, United Nations, 2015. Available at: www. un.org/sustainabledevelopment/health (Accessed: 11.02.2018).

2. UNAIDS. 90-90-90; An ambitious treatment target to help end the AIDS epidemic. $1^{\text {st }}$ ed. Geneva: Joint United Nations Programme on HIV/AIDS; 2014. 
3. NSACP. Annual Report - 2018. Colombo, Sri Lanka: National STD/AIDS Control Programme; 2019.

4. AIDS info. U.S. Department of Health and Human Services. [Online] 2016. Available at: https://aidsinfo.nih.gov/understanding-hiv-aids/ fact-sheets/19/47/hiv-testing (Accessed: 09.02.2018).

5. Greenwald LJ, Burstein GR, Pincus J, Branson B. A rapid review of rapid HIV antibody tests. Curr Infect Dis Rep 2006; 8: 125-131.

6. Mabuto T, Latka MH, Kuwane B, Churchyard GJ, Charalambous S, Hoffmann ChJ. Four models of HIV counseling and testing: utilization and test results in South Africa. PLoS One 2014; 9: e102267.

7. Rice JD, Johnson BA, Strawderman RL. Modeling the rate of HIV testing from repeated binary data amidst potential never-testers. Oxford University Press; 2018.

8. Suchira Suranga M, Karawita DA, Bandara S, Rajakaruna R. Factors associated with clinic escorts in peer-led HIV prevention interventions for men who have sex. J Virus Erad 2016; 2 (Suppl 4): 41-44.

9. Suchira Suranga M, Karawita DA, Sugath Bandara A, Kumara Rajakaruna D. Time taken to escort men who have sex with men (MSM) for HIV testing in the peer group interventions in Sri Lanka. Sri Lanka Journal of Sexual Health and HIV Medicine 2016; 2: 27-34.

10. NSACP. Sri Lanka National HIV Monitoring and Evaluation Plan (20162022). Colombo: National STD/AIDS Control Programme; 2017.

11. The Family Planning Association of Sri Lanka. Monitoring and Evaluation Policy. Colombo; 2013.

12. Smith T, Smith B. Kaplan Meier And Cox Proportional Hazards Modeling: Hands On Survival Analysis. San Diego, CA: Department of Defense Center for Deployment Health Research, Naval Health Research Center; 2003.

13. Kelkile D. Statistical analysis of adult HIV/AIDS patients and modelling of AIDS disease progression. Science Journal of Applied Mathematics and Statistics 2016; 4: 189-201.

14. Tekle G. Modeling time to death of HIV infected patients on antiretroviral therapy in case of Hossana Queen Elleni Mohammad Memorial Hospital, South Ethiopia. Journal of Health, Medicine and Nursing 2017; 37: 1-13.

15. Borucka J. Extension of Cox model for non proportional hazard purpose. Ekonometria Econometrics 2014; 3: 85-101.

16. Hosmer WD, Lemeshow S, May S. Applied Survival Analysis: Regression Modeling of Time-to-Event Data. Wiley; 2008.

17. NSACP. National size estimation of most-at-risk populations (MARPs) for HIV in Sri Lanka. National STD/AIDS Control Programme, Ministry of Health. Colombo: National STD/AIDS Control Programme; 2018.

18. National HIV Testing Guideline. Sri Lanka. Colombo: National STD and AIDS Control Programme; 2016.

19. Sri Lanka National HIV Prevention Strategic Plan; 2018-2022. Colombo: National STD/AIDS Control Programme; 2017.

20. National HIV Strategic Plan; 2013-2017. Colombo: National STD/ AIDS Control Programme; 2013. 